\title{
TREINO MODERADO DE CORRIDA CAUSA ALTERAÇÕES NAS PROPRIEDADES BIOMECÂNICAS DO TENDÃO DO CALCÂNEO?!
}

\author{
MS. RODRIGO RIBEIRO DE OLIVEIRA \\ Faculdade de Medicina, Universidade Federal do Ceará \\ (Fortaleza - Ceará - Brasil) \\ E-mail: rodrigo@ufc.br
}

\section{MS. MÁRCIO ALMEIDA BEZERRA}

Bacharelado em Fisioterapia, Faculdade de Integração do Sertão; Litoral Serviços Médicos

(Recife - Pernambuco - Brasil)

E-mail: marcioalmeidabezerra@gmail.com

\section{GRAD. PATRÍCIA VERÇOZA DE CASTRO SILVEIRA \\ Centro de Ciências Biológicas, Universidade Federal de Pernambuco \\ (Recife - Pernambuco - Brasil) \\ E-mail: pativercoza@hotmail.com}

\section{GABRIEL NUNES DE MESQUITA}

Curso de Fisioterapia, Universidade Federal de Pernambuco

(Recife - Pernambuco - Brasil)

E-mail: gabriel_aiki@hotmail.com

\section{MS. MAGNO FELIPE HOLANDA BARBOZA INÁCIO TEIXEIRA \\ Programa de Pós-Graduação em Engenharia Química, \\ Universidade Federal de Pernambuco (Recife - Pernambuco - Brasil) \\ E-mail:maxd@bol.com.br}

I. O estudo foi apoiado pela CAPES (Coordenação de Aperfeiçoamento de Pessoal de Nível Superior). O estudo foi desenvolvido a partir de recursos provenientes do projeto de pesquisa CNPq/Universal n 477096/2008-5. Agradecemos aos conhecimentos compartilhados e a colaboração da Prof. ${ }^{a}$ Yêda Medeiros Bastos de Almeida do Laboratório de Materiais Poliméricos e Caracterização do Departamento de Engenharia Química da UFPE e do Prof. Daniel Lambertz do Laboratório de Biomecânica do Departamento de Nutrição da UFPE. 


\section{DRA. SILVIA REGINA ARRUDA DE MORAES}

Programa de Pós-Graduação em Fisioterapia, Universidade Federal de Pernambuco

(Recife - Pernambuco - Brasil)

E-mail: silvicola62@yahoo.com.br

\section{RESUMO}

O efeito do treinamento aeróbio ainda apresenta resultados conflitantes com relação às respostas biomecânicas apresentadas pelo tendão após um período de treinamento aeróbio moderado, como a corrida. Este estudo tem como objetivo avaliar o efeito de um protocolo de corrida de intensidade moderada em esteira sobre as propriedades mecânicas dos tendões do calcâneo de ratos. Para o ensaio mecânico os tendões foram tracionados até o ponto de fracasso do espécime. $O$ treinamento aeróbiode intensidade moderada em ratos não causa alterações nas propriedades biomecânicas do tendão de calcâneo, desta forma, parece não representar risco podendo ser eficaz por não levar o tendão do Calcâneo ao estresse e a fadiga mecânica.

PALAVRAS CHAVE: Treinamento aeróbio moderado; tendão do calcâneo; propriedades mecânicas; sistema musculoesquelético.

\section{INTRODUÇÃO}

A alteração estrutural do tendão do calcâneo é muito comum em atletas, bem como na população em geral (PAAVOLA et al., 2002). Nas últimas décadas ocorreu um crescente aumento da prática do atletismo competitivo ou por lazer, promovendo um acréscimo na incidência de lesões no tendão do calcâneo (LEPPILAHTI et al., 1996; KANNUS; NATRI, I997; JARVINEN et al., 200 I).

As propriedades mecânicas do tendão do calcâneo influenciam na função e no desempenho do sistema músculo-esquelético durante a locomoção de humanos (BIEWENER; ROBERTS, 2000; BOBBERT, 200I; ROBERTS; MARSH, 2003; BIEWENER et al., 2004). As características elásticas dos tendões exarcebam a eficiência do músculo durante as atividades com ciclos de alongamento-encurtamento músculo-tendinoso, como por exemplo, na corrida. Dessa forma, as características elasticas do tendão do Calcâneo reduzem o trabalho do músculo tornando o ato de correr com menor gasto energético (BIEWENER; ROBERTS, 2000; HOF et al., 2002; ROBERTS; MARSH, 2003).

Diversos estudos avaliaram as propriedades mecânicas de tendões e demonstraram sua plasticidade em responder a diferentes situações, tais como: a imobilização 
e/ ou a suspensão (ALMEIDA-SILVEIRA et al., 2000; NATALI et al., 2008), os exercícios em plataformas vibratórias (LEGERLOTZ; SCHJERLING, LANGEBERG, 2007), o alongamento (VIIDIK, 1967), o treinamento de força (SIMONSEN; KLITGAARD; BOJSEN-MOLLER, 1995; MAGNUSSON et al., 2003; SEYNNES et al., 2009) e o treinamento de endurance (VIIDIK, 1967; SIMONSEN; KLITGAARD; BOJSEN-MOLLER, I 995; BUCHANAN; MARSH, 200 I; LEGERLOTZ; SCHJERLING; LANGBERG, 2007). No caso especifico do treinamento aeróbico, apesar de já ter sido bastante estudado, os resultados ainda são conflitantes com relação às respostas biomecânicas apresentadas pelo tendão após o período de treinamento aeróbico.

Experimentalmente, em um estudo pioneiro, foi detectado incremento no módulo de elasticidade no tendão do calcâneo de ratos após exercício de corrida (VILARTA; VIDAL, 1989), no entanto, este resultado não se repetiu em outros estudos. Visto que, um estudo (HUANG, PERRY; SOSLOWSKY, 2004) não observou quaisquer alteração desta variável nos animais corredores quando comparado aos não treinados e ao compararem as propriedades mecânicas do tendão do calcâneo de ratas submetidas a duas diferentes modalidades de exercícios: plataforma vibratória, e cicloergômetro voluntário, também não observaram qualquer adaptação e/ou alteração do tendão (LEGERLOTZ; SCHJERLING; LANGBERG, 2007).

Em humanos, com a utilização o ultra-som de imagem para a determinação das propriedades mecânicas do tendão, os resultados encontrados também são discordantes. Pois, um estudo detectou aumento da rigidez nos tendões do calcâneo dos indivíduos corredores em comparação com o grupo controle (KUBO et al., 2002), em contraste, outros estudos não detectaram diferenças na rigidez do tendão do calcâneo entre os corredores e não corredores (ROSAGER et al., 2002; KARAMANIDIS; ARAMPATZIS, 2006).

Desta forma, tendo em vista a controvérsia ainda existente sobre essa temática, e o fato de que a maioria dos estudos existentes avalia a influência de diferentes formas de treinamento sobre o processo de adaptação do tendão (VIIDIK, 1967; SOMMER, 1987; VILARTA; VIDAL, I989; BIEWENER; ROBERTS, 2000; BUCHANAN; MARSH, 200I; HUANG; PERRY; SOSLOWSKY, 2004; KARAMANIDIS; ARAMPATZIS, 2006; LEGERLOTZ; SCHJERLING; LANGBERG, 2007), este estudo propos avaliar se um exercício de intensidade moderada, como a corrida, teria algum efeito sobre as propriedades biomecânicas do tendão do calcâneo de ratos. 
ANIMAIS

Foram utilizados 20 ratos albinos da linhagem Wistar (Rattus Norvegicus), machos, mantidos em gaiolas plásticas coletivas com seis animais/gaiola (4Ix34x$16 \mathrm{~cm})$ em condição controlada de temperatura $\left(23 \pm 1^{\circ} \mathrm{C}\right)$ e fotoperíodo invertido (luz/escuridão) de 12 h.

Os procedimentos realizados para manejo e cuidado dos animais estão de acordo com as normas do National Institute of Health Guide for Care and Use of Laboratory Animal e foram aprovados pela comissão de Ética em Experimentação Animal do Centro de Ciências Biológicas - UFPE, sob o protocolo $n^{\circ}$. 23076.012707/2009-27.

\section{TREINAMENTO FÍSICO}

Ao atingirem a idade de 90 dias (peso de 350 a 400g) os animais foram distribuídos aleatoriamente em dois grupos: Grupo Controle - GC $(n=1$ I) e o Grupo Treinado- GT ( $n=09)$, que foi submetido ao treinamento físico moderado contínuo em tapete ergométrico (Esteira motorizada EP I 3 I Insight). Utilizando-se uma adaptação do protocolo de Leandro, Levada, Hirabara et al. 2007 (treino I vez ao dia, 5 dias por semana durante 8 semanas com intensidade aproximada de $70 \%$ do VO2max). Na primeira semana (semana de adaptação) duração de $10 \mathrm{~min} /$ dia e velocidade máxima de $5 \mathrm{~m}$.min- I. Nas 7 semanas seguintes foi acrescentado tempo e intensidade até atingir a velocidade máxima de $13 \mathrm{~m}$.min- | e tempo máximo de 60 min. Cada sessão de treinamento, a partir da segunda semana, foi dividida em quatro estágios progressivos: (a) período de aquecimento (3 a 5 minutos); (b) zona intermediária ( 14 a 20 minutos), (c) zona de treinamento ( 0 a 30 minutos), e (d) período resfriamento - volta calma (3 a 5 minutos). De acordo como apresentado na Tabela $I$.

Durante o período de treinamento, os animais do grupo controle permaneceram em suas respectivas gaiolas. 
Tabela I. Descrição do protocolo de treinamento físico demonstrando a velocidade e a duração das sessões com a divisão das fases de treinamento

\begin{tabular}{|c|c|c|c|c|}
\hline \multirow{2}{*}{ Semanas } & \multicolumn{2}{|c|}{ Velocidade } & \multirow{2}{*}{ Duração (min) } & \\
\hline & $\mathrm{km} / \mathrm{h}$ & m.min-I & & \\
\hline \multirow{4}{*}{ Inicial (adaptação) } & 0.1 & 2 & 2 & \multirow{4}{*}{10} \\
\hline & 0.2 & 3 & 2 & \\
\hline & 0.3 & 5 & 4 & \\
\hline & 0.1 & 2 & 2 & \\
\hline \multirow{4}{*}{ Segunda semana } & 0.2 & 3 & 3 & \multirow{4}{*}{20} \\
\hline & 0.3 & 5 & 4 & \\
\hline & 0.4 & 7 & 10 & \\
\hline & 0.2 & 3 & 3 & \\
\hline \multirow{5}{*}{ Terceira semana } & 0.3 & 5 & 3 & \multirow{5}{*}{30} \\
\hline & 0.4 & 7 & 4 & \\
\hline & 0.5 & 8 & 5 & \\
\hline & 0.6 & 10 & 15 & \\
\hline & 0.3 & 5 & 3 & \\
\hline \multirow{5}{*}{ Quarta semana } & 0.4 & 7 & 3 & \multirow{5}{*}{30} \\
\hline & 0.5 & 8 & 4 & \\
\hline & 0.6 & 10 & 5 & \\
\hline & 0.7 & 12 & 15 & \\
\hline & 0.4 & 7 & 3 & \\
\hline \multirow{5}{*}{ Quinta semana } & 0.4 & 7 & 3 & \multirow{5}{*}{40} \\
\hline & 0.5 & 8 & 7 & \\
\hline & 0.6 & 10 & 7 & \\
\hline & 0.7 & 12 & 20 & \\
\hline & 0.4 & 7 & 3 & \\
\hline \multirow{5}{*}{ Sexta semana } & 0.4 & 7 & 5 & \multirow{5}{*}{50} \\
\hline & 0.5 & 8 & 7 & \\
\hline & 0.6 & 10 & 8 & \\
\hline & 0.7 & 12 & 25 & \\
\hline & 0.4 & 7 & 5 & \\
\hline \multirow{5}{*}{ Sétima semana } & 0.5 & 8 & 5 & \multirow{5}{*}{50} \\
\hline & 0.6 & 10 & 7 & \\
\hline & 0.7 & 12 & 8 & \\
\hline & 0.8 & 13 & 25 & \\
\hline & 0.5 & 8 & 5 & \\
\hline \multirow{5}{*}{ Oitava semana } & 0.5 & 8 & 5 & \multirow{5}{*}{60} \\
\hline & 0.6 & 10 & 8 & \\
\hline & 0.7 & 12 & 12 & \\
\hline & 0.8 & 13 & 30 & \\
\hline & 0.5 & 8 & 5 & \\
\hline
\end{tabular}


Após o término do treinamento os animais dos dois grupos foram anestesiados, por um pesquisador experiente e devidamente treinado, com solução de Xilazina (Rompum ${ }^{\circledR}$ - Bayer) ( I Omg/Kg) e Cloridrato de Ketamina (Ketalar $\left.{ }^{\circledR}\right)(25 \mathrm{mg} /$ $\mathrm{kg}), 0,10 \mathrm{ml}$ para cada $100 \mathrm{~g}$ de peso e realizada uma incisão na pele da região posterior da pata esquerda para desinserção dos tendões do calcâneo em suas origens e inserções. Após a coleta o tendão de foi recoberto com gaze umedecida com solução salina 9\% e encaminhado para análise do ensaio mecânico convencional.

\section{ENSAIO MECÂNICO}

Para o ensaio mecânico o tendão foi alojado a um conector de metal $(2,5 \times$ $3,5 \mathrm{~cm}$ ) para cada extremidade e fixado em uma máquina de ensaio mecânico convencional (EMIC, modelo DL 500, Brasil) através de garras auto travantes por efeito de alavanca (FIGURA I). Os tendões foram tracionados até o ponto de fracasso do espécime, em velocidade de $0,1 \mathrm{~mm} / \mathrm{s}$, com célula de carga de $500 \mathrm{~N}$ (EMIC, modelo SV500, Brasil). Os parâmetros avaliados foram: Módulo Elástico (MPa), Tensão Força Máxima (MPa), Deformação Específica Força Máxima (mm), Energia/Área (N.mm/ $\left.\mathrm{mm}^{2}\right)$, Força/largura na força máxima $(\mathrm{N} / \mathrm{mm})$ e Área de secção transversa $\left(\mathrm{mm}^{2}\right)$.

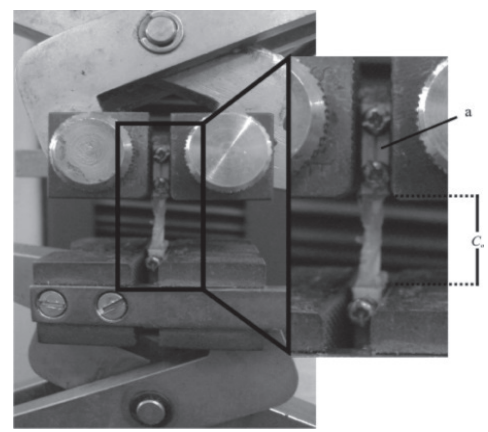

Figura I. Máquina de ensaio mecânico com o tendão do calcâneo fixado nas garras auto-travantes. $\mathrm{A}=$ Conector de metal. $\mathrm{CO}=$ Comprimento inicial.

A área de secção transversa (AST) para o ensaio mecânico convencional foi estimada de acordo com a fórmula da determinação da área da elipse $A S T=\left(\frac{D . T}{4}\right) \pi$ $A S T=\left(\frac{D . T}{4}\right) \pi$, sendo o $\mathrm{D}=$ é a largura do terço médio do tendão, $\mathrm{T}=\mathrm{a}$ espessura do terç̧o médio do tendão. Sendo essa forma geométrica mais aproximada da observada nos cortes transversais do tendão (SILVEIRA; NERY, I999).

\section{ANÁLISE ESTATIISTICA}

Para descrever as características da amostra foram utilizadas medidas descritivas, tais como: medida de tendência central (média) e dispersão (desvio padrão). 
Para determinar se a distribuição de probabilidade subjacente difere da normalidade foi aplicado o teste de Kolmolgorov-Smirnov, e o mesmo acusou padrão de distribuição normal. Desta forma, para comparação das médias das variáveis numéricas entre os vários tratamentos empregados foi utilizado o teste $T$ para amostras independentes (Independent-Samples T Test) para comparação entre os dois grupos. Os dados foram analisados no software SPSS (Statistical Package for Social Sciences) e admitido nível de significância de $1 \%$.

\section{RESULTADOS}

No dia da coleta do material o peso corporal dos animais foi de 437.4 (I 6.34) g para o grupo controle e de 443,8 ( I3,45) g para o grupo induzido ao treinamento aeróbio demonstrando não haver diferença do peso corpóreo entre os dois grupos avaliados $(p=0,475)$.

A avaliação das propriedades biomecânicas do tendão do calcâneo demonstrou que o módulo elástico (MPa), a tensão da força máxima (MPa), a deformação específica (\%), a deformação da força máxima ( $\mathrm{mm}$ ), a energia/área ( $\left.\mathrm{N} \cdot \mathrm{mm} / \mathrm{mm}^{2}\right)$ e a área de secção transversa $\left(\mathrm{mm}^{2}\right)$ não apresentaram diferenças entre os animais dos dois grupos avaliados.
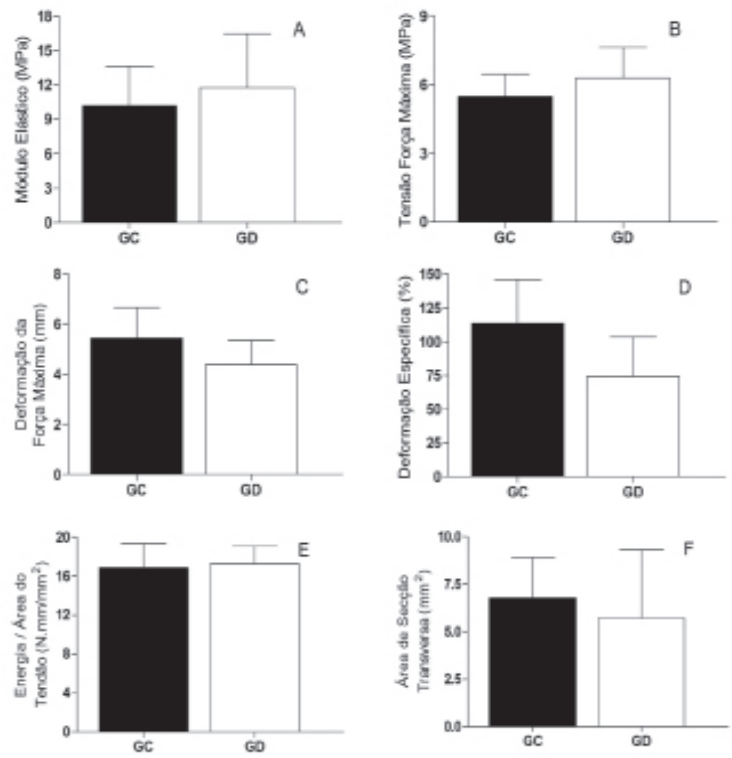

Figura 2. Efeito do treinamento aeróbio moderado durante oito semanas sobre as propriedades do tendão do calcâneo de ratos. Os Valores estão expressos em média e desvio padrão. Não houve diferença estatística entre os grupos nos gráficos A, B, C, D, E e F - p>0,0 I (Independent-Samples T Test).

$\mathrm{GC}=$ Grupo controle; GT = Grupo treinado. 


\section{DISCUSSÃO}

tendão do calcâneo apresenta uma grande importância funcional na mecânica da locomoção, em especial na corrida (LICHTWARK; BARCLAY, 20 I0). Todavia, ainda há controvérsia sobre o conhecimento da resposta biomecânica do tendão, quando submetido ao treinamento aeróbiomoderado.

Nesse estudo o treinamento de corrida em intensidade moderada, durante oito semanas não alterou as propriedades mecânicas dos tendões dos calcâneos de ratos quando submetidos ao ensaio mecânico de tração.

O método de análise das propriedades mecânicas do tendão utilizado foi similar aos empregados nos estudos de Almeida-Silveira et al. (2000) e de Reddy et al. (2002). Tendo em vista que as propriedades mecânicas de tecidos biológicos podem variar de acordo com o tempo de vida e com a espécie de mamífero avaliado, este protocolo, comparou das médias obtidas nos ensaios mecânicos dos grupos do presente estudo (GC e GT) e não extrapolou a comparação com os valores absolutos disponíveis na literatura.

Estudos que optaram por protocolos de treinamento com período de maior duração demonstraram diferenças nas propriedades mecânicas dos tendões, observando adaptação do tendão do tibial posterior, do tendão fibular e do tendão do calcâneo de coelhos que foram submetidos à corrida com protocolo com duração de 40 semanas, demonstrando um aumento de 10\% na rigidez (stiffness) dos tendões do calcâneo e do tibial posterior e aumento de 5\% da resistência à tração dos mesmos tendões (VIIDIK, 1967; VIIDIK et al., 1982). No entanto, estes estudos não descrevem a intensidade do protocolo de corrida aplicado.

Outro estudo submeteu suínos a um programa de corrida (velocidade máxima de 2,2 m/s durante 12 semanas) e demonstrou um incremento no stiffness e aumento da força máxima em $62 \%$ no tendão dos extensores digitais. No entanto, o treinamento não alterou as propriedades do tendão dos flexores digitais. Todavia, o tendão dos extensores digitais suportou menores cargas ao teste de tração quando comparado ao tendão dos flexores digitais (WOO et al., 1980). Os resultados deste estudo podem ser indicativos que as alterações na capacidade visco-elásticas e a redução das propriedades mecânicas do tendão, em resposta ao treinamento aeróbico, poderão predispor a dano precocemente, quando exposto ao estresse de alta tensão.

Entretanto, semelhante aos resultados desse estudo, a avaliação do tendão patelar de camundongos submetidos ao treinamento aeróbico, em esteira, demonstrou que a força de tração (ponto de fracasso) dos tendões de animais treinados durante sete semanas, não diferiu dos animais não corredores (KIISKINEN, 1977). 
tendão pode ser danificado também por um único macro-trauma e/ou uma grande tensão, ou por repetições de micro-traumas. Para avaliar este aspecto, Schechtman e Bader (1997) simularam um padrão, in vivo, de estresse cíclico ao tendão do extensor longo dos dedos com 20\% da tensão de ruptura (máxima), e a insuficiência do tendão ocorreu em cerca de 300.000 ciclos, o que equivaleria aproximadamente a quatro meses de caminhada normal. A carga cíclica trará uma resposta adaptativa ao tendão do calcâneo e poderá servir como um mecanismo protetor aos os danos associados a cargas repetidas, no entanto, o aumento expressivo do número de ciclos levará a lesão e/ou ao fracasso do tendão. Desta forma, acredita-se que o aumento nas propriedades mecânicas, tais como, o incremento do stiffness, permitirá menor extensibilidade do tendão, o que pode resultar em menores danos estruturais.

No entanto, o presente estudo demonstra que a corrida de intensidade e duração moderados, logo com menor quantidade de ciclos, não promoveu alterações nas propriedades mecânicas, o que pode ser interpretado como a ausência de necessidade de responder ao estresse e a fadiga mecânica.

O tendão sofre deformação elástica na sua estrutura mecânicas para incrementar o armazenamento de energia elástica e, posteriormente, liberá-la como um mecanismo para maximixar a locomoção com gasto energêtico econômico e menor dano estrutural (ROBERTS et al., 1997), contudo, o incremento no armazenamento de energia elástica não dependerá das propriedades mecânicas, a exemplo disso, Kubo et al. (2002) observaram as propridades mecânicas significativamente mais elevadas no tendão do músculo vasto lateral de corredores fundistas em relação aos não corredores. No entanto, ao realizarem movimentos de salto foi revelado um menor potencial de armazenamento de energia elástica nos corredores.

O aumento das propriedades mecânicas do tendão exposto ao treinamento aeróbio de longa duração e alta intensidade não está associado com a exigência de suportar maior carga, mas representa um mecanismo de resistência do tendão ao dano proveniente da fadiga mecânica (SIMONSEN; KLITGAARD; BOJSENMOLLER, 1995). Assim, a atividade deverá ter intensidade e frequência suficiente para adaptação do tecido, mas que não leve a fadiga do mesmo.

Para analisar as propriedades mecânicas de tendões do calcâneo, foi utilizado ensaio de tração mecânica convencional realizado em uma única estirpe, por conseguinte, os componentes de visco-elásticas dos tendões não pôde ser quantificada separadamente. A análise in vitro não refletem aspectos importantes, tais como o ponto de inflexão da inserção do tendão no osso calcâneo e não apresenta a atividade do grupameto muscular do tríceps sural. Outras pesquisas devem ser realizadas de forma longitudinal para que se possa descrever uma relação de causa/ efeito da atividade física e possiveis alterações mecânicas nos tendões do calcâneo. 


\section{CONCLUSÃO}

O treinamento aeróbio de intensidade moderada em ratos não causa alterações nas propriedades biomecânicas do tendão de calcâneo. A corrida de intensidade e de frequência moderadas parece não representar riscos ao tendão do calcâneo podendo ser indicada por não levar a estrutura do tendão do calcâneo ao estresse e a fadiga mecânica.

\section{Do Moderate Running Training Changes the Biomechanical Properties of the Achilles Tendon?}

ABSTRACT: The effect of aerobic training has presented conflicting results regarding the biomechanical responses submitted by the tendon after a period of moderate aerobic training such as running. This study aims to evaluate the effect of a protocol running on a treadmill at moderate intensity on the mechanical properties of the Achilles tendons of rats. For mechanical test the tendons were pulled to the point of failure of the specimen. The moderate-intensity aerobic training in rats does not changes in the biomechanical properties of the Achilles tendon, thus, does not seem to pose a risk can be effective for not taking the Achilles tendon to mechanical stress and fatigue.

KEYWORDS: Moderate aerobic training; achilles tendon; mechanical propertie; musculoskeletal system.

\section{¿Entrenamiento físico moderado causan cambios en las propiedades biomecánicas del tendón de Aquiles?}

RESUMEN: El efecto de entrenamiento aerobio ha presentado resultados contradictorios respecto a las respuestas biomecánicas presentado por el tendón después de un período de entrenamiento aerobio moderado, como correr. Este estudio pretende evaluar el efecto de un protocolo de correr en una cinta a una intensidad moderada en las propiedades mecánicas de los tendones de Aquiles de las ratas. Para pruebas mecánicas de los tendones se retiraron hasta el punto de fracaso de la muestra. El entrenamiento aerobio de intensidad moderada en las ratas no provoca cambios en las propiedades biomecánicas del tendón de Aquiles, por lo tanto, no parece representar un riesgo puede ser eficaz para no tomar el tendón de Aquiles a la tensión mecánica y la fatiga.

PALABRAS CLAVE: Entrenamiento aerobio moderado; del tendón de Aquiles; las propiedades mecánicas; sistema musculoesquelético. 


\section{REFERÊNCIAS}

ALMEIDA-SILVEIRA, M. I. et al. Changes in stiffness induced by hindlimb suspension in rat Achilles tendon. European Journal of Applied Physiology, Heidelberg, v. 8I, n. 3, p. 252-257, feb. 2000.

BIEWENER, A. A.; ROBERTS, T. J. Muscle and tendon contributions to force, work, and elastic energy savings: A comparative perspective. Exercise and Sport Sciences Reviews, Hagerstown, v. 28, n. 3, p. 99- I07, jul. 2000.

BIEWENER, A. A. et al. Muscle mechanical advantage of human walking and running: Implications for energy cost. Journal of Applied Physiology, Bethesda, v. 97, n. 6, p. 2266-2274, dec. 2004.

BOBBERT, M. F. Dependence of human squat jump performance on the series elastic compliance of the triceps surae: a simulation study. Journal of Experimental Biology, Cambridge, v. 204, pt. 3, p. 533-542, feb. 2001.

BUCHANAN, C. I.; MARSH, R. L. Effects of long-term exercise on the biomechanical properties of the Achilles tendon of guinea fowl. Journal of Applied Physiology, Bethesda, v. 90, n. I, p. |64-17|, jan. 2001.

HOF, A. L. et al. Mechanics of human triceps surae muscle in walking, running and jumping. Acta Physiologica Scandinavica, Oxford, v. 174, n. I, p. 17-30, jan. 2002.

HUANG, T. F. ; PERRY, S. M. ; SOSLOWSKY, L. J. The effect of overuse activity on Achilles tendon in an animal model: a biomechanical study. Annals of Biomedical Engineering, New York, v. 32, n. 3, p. 336-34I, mar. 2004.

JARVINEN, T. A. et al. Achilles tendon injuries. Current Opinion in Rheumatology, Philadelphia, v. 13, n. 2, p. I50-155, mar. 200।.

KANNUS, P.; NATRI, A. Etiology and pathophysiology of tendon ruptures in sports. Scandinavian Journal of Medicine and Science in Sports, Copenhagen, v. 7, n. 2, p. $107-1$ I 2, apr. 1997.

KARAMANIDIS, K.; ARAMPATZIS, A. Mechanical and morphological properties of human quadriceps femoris and triceps surae muscle-tendon unit in relation to aging and running. Journal of Biomechanics, New York, v. 39, n. 3, p. 406-417, june 2006.

KIISKINEN, A physical training and connective tissues in young mice: physical properties of Achilles tendons and long bones. Growth, Lakeland, v. 4I, n. 2, p. 123, june 1977.

$\mathrm{KUBO}, \mathrm{K}$. et al. Effects of resistance and stretching training programmes on the viscoelastic properties of human tendon structures in Vivo. Journal of Physiology, Paris, v. 538, n. Pt I, p. 219-226, jan. 2002.

LEGERLOTZ, K. et al. The effect of running, strength, and vibration strength training on the mechanical, morphological, and biochemical properties of the Achilles tendon in rats. Journal of Applied Physiology, Bethesda, v. 102, n. 2, p. 564-572, feb. 2007. 
LEPPILAHTI, J. et al. Incidence of Achilles tendon rupture. Acta Orthopaedica Scandinavica, Copenhagen, v. 67, n. 3, p. 277-279, june 1996.LICHTWARK, G. A.; BARCLAY, C. J. The influence of tendon compliance on muscle power output and efficiency during cyclic contractions. Journal of Experimental Biology, Cambridge, v. 213, n. 5, p. 707, mar. 2010.

MAGNUSSON, S. P. et al. Tendon properties in relation to muscular activity and physical training. Scandinavian Journal of Medicine and Science in Sports, Copenhagen, v. 13, n. 4, p. 211-223, aug. 2003.

NATALI, L. H. et al. Efeitos da corrida em esteira em músculos sóleos de ratos encurtados por imobilização. Revista Brasileira de Medicina do Esporte, São Paulo, v. I4, n. 6, p. 490493, nov. 2008.

PAAVOLA, M. et al. Achilles tendinopathy. Journal of Bone and Joint Surgery, Boston, v. 84-A, n. I I, p. 2062-2076, nov. 2002.

REDDY, G. K.; STEHNO-BITTEL, L.; ENWEMEKA, C. S. Glycation-induced matrix stability in the rabbit achilles tendon. Archives of Biochemistry and Biophysics, New York, v. 399, n. 2, p. 174-180, mar. 2002.

ROBERTS, T. J.; MARSH, R. L. Probing the limits to muscle-powered accelerations: lessons from Jumping bullfrogs. Journal of Experimental Biology, Cambridge, v. 206, pt. I 5, p. 25672580, aug. 2003.

ROBERTS, T. J. et al. Muscular force in running turkeys: the economy of minimizing work. Science, New York, v. 275, n. 5303, p. I I I3-। I I 5, feb. 1997.

ROSAGER, S. et al. Load displacement properties of the human triceps surae aponeurosis and tendon in runners and non runners. Scandinavian Journal of Medicine \& Science in Sports, Copenhagen, v. 12, n. 2, p. 90-98, apr. 2002.

SCHECHTMAN, H.; BADER, D. L. In vitro fatigue of human tendons. Journal of Biomechanics, New York, v. 30, n. 8, p. 829-835, aug. 1997.

SEYNNES, O. R. et al. Training-induced changes in structural and mechanical properties of the patellar tendon are related to muscle hypertrophy but not to strength gains. Journal of Applied Physiology, Bethesda, v. 107, n. 2, p. 523-530, may 2009.

SILVEIRA, A. C. M.; NERY, C. A. S. Macroscopic and morphometric study of the posterior tibial tendon. Revista Brasileira de Ortopedia, São Paulo, v. 34, n. 8, p. 475-480, aug. 1999.

SIMONSEN, E. B.; KLITGAARD, H.; BOJSEN-MOLLER, F. The influence of strength training, swim training and ageing on the achilles tendon and $\mathrm{m}$. Soleus of the rat. Journal of Sports Sciences, London, v. 13, n. 4, p. 291-295, aug. 1995.

SOMMER, H. M. The biomechanical and metabolic effects of a running regime on the Achilles tendon in the rat. International Orthopaedics, Berlin, v. II, n. I, p. 7I-75, jan. 1987. 
VIIDIK, A. The effect of training on the tensile strength of isolated rabbit tendons. Scndinavian Journal of Plastic and Reconstructive Surgery and Hand Surgery, Stockholm, v. I, n. 2, p. I4I|47, feb. 1967.

VIIDIK, A. et al. On fundamental and phenomenological models, structure and mechanical properties of collagen, elastin and glycosaminoglycan complexes. Biorheology, Elmsford, v. 19, n. 3, p. 437-45I, aug. 1982.

VILARTA, R.; VIDAL, C. Anisotropic and biomechanical properties of tendons modified by exercise and denervation: aggregation and macromolecular order in collagen bundles. Matrix, Stuttgart, v. 9, n. I, p. 55-61, jan. 1989.

WOO, S. L. et al. The biomechanical and biochemical properties of swine tendons-long term effects of exercise on the digital extensors. Connective Tissue Research, New York, v. 7, n. 3, p. 177-183, aug. 1980.

Recebido em: 02 fev. 2012

Aprovado em: 03 jul. 2012

Endereço para correspondência

Rodrigo Ribeiro de Oliveira

Rua Alexandre Baraúna 949

Bairro Rodolfo Teófilo

Fortaleza / CE

CEP: $60430-160$ 